\title{
Associative transfer as a function of conceptual similarity of stimuli in pre-school aged children'
}

LOUIS E. PRICE, THE UNIVERSITY OF NEW MEXICO ANNE C. SARAVO, FELS RESEARCH INSTITUTE

The effects of conceptual similarity on the $A-C$ and $A-B r$ transfer paradigms were investigated in pre-school aged children using pictures of familiar objects as stimuli. The effect of similarity was significant in both Task 1 and Task 2 as was performance over trials. Significant negative transfer was found in the second task for both the $A-C$ and $A-B r$ paradigms. The extent of this interference was not a function of stimulus similarity.

Underwood, Ekstrand, \& Keppel (1965) have shown that conceptual similarity retards paired associate learning, a finding which is consistent with the notion that associative interference will occur among conceptually related terms in a paired associate list. However, although negative transfer effects are generally interpreted in terms of associative interference, the effects of stimulus similarity on transfer paradigms have not been conclusively determined. The two paradigms which generally result in negative transfer are the $\mathrm{A}-\mathrm{C}$ and $\mathrm{A}-\mathrm{Br}$ paradigms, with interference more pronounced in the latter (e.g., Besch \& Reynolds, 1958). In a previous study by Saravo \& Price (1967), some evidence was found for increasing interference in both the A-C and $\mathrm{A}-\mathrm{Br}$ paradigms with increases in stimulus similarity. The failure of the main effect for similarity to reach significance and the interaction of counterbalancing forms with similarity and paradigms, however, made a clear-cut interpretation of this relationship difficult.

The purpose of the present study was to determine whether variations in conceptual similarity would affect the amount of negative transfer produced by the $\mathrm{A}-\mathrm{C}$ and A-Br paradigms. Pre-school age children were used as Ss in order to extend the findings of other investigations of paired associate transfer which used adult Ss. Subjects

Seventy-two children between three and five years of age were selected from the nursery school and the day care nursery at Westover Air Force Base, Chicopee, Massachusetts and the University of Massachusetts nursery school, Amherst, Massachusetts.

\section{Apparatus and Stimuli}

Stimuli and responses were pictures of familiar objects pasted on white plastic cards for presentation on a Hunter Card Master. Stimuli in the high similarity condition consisted of pictures of different breeds of dogs, those in the medium similarity condition were pictures of barnyard animals, and pictures of unrelated familiar objects comprised the stimuli of the low simi- larity condition. Response members were also pictures of unrelated objects. Exact duplicates of the response members for both tasks were displayed directly below the aperture of the Card Master on a large cardboard square.

The Ss were assigned to one of three groups differing in degree of stimulus similarity: high, medium, and low. Within each of these groups, half the Ss learned two successive four-pair lists with two pairs each corresponding to the A-C and C-D paradigms while the remaining Ss learned two four-pair lists with two pairs each representing the $\mathrm{A}-\mathrm{Br}$ and $\mathrm{C}-\mathrm{D}$ paradigms. Within each of these six groups, Ss were assigned to one of two subgroups in order to counterbalance for possible differential difficulty of the two pairs representing the experimental and control paradigms.

\section{Procedure}

One month before any Ss were given the learning tasks, familiarization trials were effected by exposing all stimulus and then response members twice in succession, asking Ss to name the objects. The familiarization trials provided a check on the familiarity and difficulty of the stimuli; however, no differences in difficulty were observed.

Each S learned two four-pair lists in succession. During both lists the anticipation interval, joint presentation interval, and interstimulus interval were 5-, $5-$, and 3-sec, respectively. The intertrial interval was 13-sec. After completing five trials on the first list, Ss were presented with the second list for five trials. Three different orders of each list were given to minimize serial associations. Correct responses, either pointing to or naming the appropriate experimenter-supplied response, were rewarded by a cinnamon candy.

\section{Results and Discussion}

Acquisition list. An analysis of variance with similarity, experimental paradigms, and counterbalancing forms as between-Ss variables and trials as a within-Ss comparison was performed on the number of correct responses given in the acquisition list. This analysis indicated a significant decrement in performance as stimulus similarity increased ( $F=8.49$, df $=2 / 66, p<$ $.001)$. Individual tests of the differences among means showed the condition of high similarity to be significantly inferior to both those of medium and low similarity $(F=8.03, \mathrm{df}=1 / 66, \mathrm{p}<.001$ and $\mathrm{F}=16.06$, $\mathrm{df}=1 / 66, p<.001$, respectively). Performance over trials was shown to improve reliably $(F=14.79$, df 
Table 1. Means and SDs for Correct Responses in the Transfer List.

Paradigms

\begin{tabular}{|c|c|c|c|c|c|c|c|c|}
\hline \multirow[b]{2}{*}{ Similarity } & \multicolumn{2}{|c|}{$\mathrm{A}-\mathrm{C}$} & \multicolumn{2}{|c|}{ C-D } & \multicolumn{2}{|c|}{$\mathrm{A}-\mathrm{Br}$} & \multicolumn{2}{|c|}{ C-D } \\
\hline & $\overline{\mathrm{M}}$ & $\mathrm{SD}$ & $\overline{\mathbf{M}}$ & $\overline{\mathrm{SD}}$ & $\overline{\mathbf{M}}$ & $\overline{\mathrm{SD}}$ & $\overline{\mathrm{M}}$ & $\overline{\mathrm{SD}}$ \\
\hline Low & $\begin{array}{l}2.92 \\
t(11)\end{array}$ & $\begin{array}{c}2.43 \\
=2.60\end{array}$ & $\begin{array}{l}4.25 \\
* *\end{array}$ & 3.57 & $\begin{array}{l}3.08 \\
\mathrm{t}(11)\end{array}$ & $\begin{array}{c}2.39 \\
=2.47\end{array}$ & $\begin{array}{c}5.42 \\
* *\end{array}$ & 3.26 \\
\hline Med & $\begin{array}{l}2.75 \\
\mathrm{t}(11)\end{array}$ & $\begin{aligned} & 1.96 \\
= & 2.33\end{aligned}$ & $\begin{array}{l}4.50 \\
* *\end{array}$ & 3.15 & $\begin{array}{l}3.50 \\
\mathrm{t}(11)\end{array}$ & $\begin{array}{c}2.84 \\
=1.84^{\circ}\end{array}$ & ${ }^{5} .08$ & 2.19 \\
\hline High & $\begin{array}{l}1.50 \\
\mathrm{t}(11)\end{array}$ & $\begin{aligned} & 1.24 \\
= & 1.68\end{aligned}$ & 2.25 & 1.60 & $\begin{array}{l}1.42 \\
t(11)\end{array}$ & $\begin{aligned} & 1.38 \\
= & 2.78\end{aligned}$ & $\begin{array}{l}3.67 \\
* * *\end{array}$ & 2.42 \\
\hline
\end{tabular}

$$
\begin{aligned}
* * * \mathrm{p} & <.01 \\
{ }^{* *} \mathrm{p} & <.025 \\
* \underline{\mathrm{p}} & <.05
\end{aligned}
$$

$=4 / 264, p<.001)$. No interactions or any other main effects reached significance $(p>.25)$.

Transfer list. The number of correct responses given in the transfer list were subjected to an analysis of variance which was similar to that performed on the firstlist data with the addition of transfer, the comparison of each experimental paradigm with the C-D control, as a within-Ss variable. Since a preliminary analysis showed neither counterbalancing forms nor trials entered into any significant interactions, the analysis was collapsed over these two variables. The effect of increasing similarity was to retard learning; the main effect for this variable proved significant beyond the .01 level $(F=5.35, d f=2 / 66)$. The effect for similarity was again produced by the high similarity condition since individual comparisons between means showed the high similarity condition to differ significantly from that of medium similarity $(F=8.21, d f=1 / 66, p<.01)$, while performance under the condition of medium similarity was not shown to be reliably different from that under the condition of low similarity $(F=.005, \mathrm{df}=1 / 66, \mathrm{p}$ $>$.20). The amount of negative transfer was highly significant $(F=30.21, \mathrm{df}=1 / 66, \mathrm{p}<.001)$, with both the $\mathrm{A}-\mathrm{C}$ and $\mathrm{A}-\mathrm{Br}$ paradigms showing poorer performance than the $C-D$ control. Individual comparisons at each level of similarity showed significant amounts of negative transfer for both the $\mathrm{A}-\mathrm{C}$ and $\mathrm{A}-\mathrm{Br}$ paradigms in comparison with the $C-D$ control with one exception, that of the A-C paradigm under high similarity (see Table 1). Performance over trials increased for all groups, significant beyond the .01 level. No other main effects or interactions reached significance.

In agreement with the findings of Underwood, Ekstrand, and Keppel, conceptual similarity was shown to retard paired associates learning; although it was not found to differentially affect transfer paradigms as was suggested in the results of the Saravo and Price study. The failure to confirm the interaction of similarity with paradigms is in line with a recent study by Price, Cobb, \& Morin (in press) in which similarity did not produce differential amounts of interference under the $\mathrm{A}-\mathrm{C}$ and $\mathrm{A}-\mathrm{Br}$ paradigms although performance on the transfer task was significantly lowered by the condition of high similarity. The additive effects of similarity and transfer paradigms suggest some modification of the theory that associative interference is due to competition from previously correct responses and generalized secondtask responses (Besch \& Reynolds, 1958). Accordingly, interference should be greater in the $\mathrm{A}-\mathrm{Br}$ paradigm since only here are the previously correct responses additionally strengthened through generalization in the second task. Interference should thus be a direct function of stimulus similarity, the difference between paradigms increasing with increases in similarity. This interaction, however, was not obtained.

Conceptual similarity may have retarded learning through the evocation of highly similar or identical covert mediating responses. If the category name, i.e., dog, were given to each stimulus in the high similarity condition, different overt responses would have to be learned, in part, to the same stimulus, albeit a covert mediating response. The finding of no difference between conditions of medium and low similarity could be due to a tendency on the part of Ss to apply the specific labels for objects in the former condition rather than the category name, barnyard animals. If conceptual similarity did indeed exert its effect in this way, this study questions the generality of the mediational deficiency hypothesis (see Reese, 1962) which asserts that covert mediating responses, particularly of a verbal nature, do not effectively influence the overt behavior of pre-school age children.

\section{References}

BESCH, N. F., \& REYNOLDS, W. F. Associative interference in verbal paired-associate learning. $J$. exp. Psychol., 1958, 55, 554-558.

PRICE, L. E., COBB, N. J., \& MORIN, N. J. Associative interference as a function of amount of first-task practice and stimulus similarity. J. exp. Child Psychol, in press.

REESE, H. W. Verbal mediation as a function of age level. Psychol. Bull, 1962, 59, 502-509.

SARAVO, A. C., \& PRICE, L. E. Associative transfer in verbal pairedassociate learning as a function of stimulus similarity. Psychon. Sci., $1967,8,315-316$.

UNDERWOOD, B. J., EKSTRAND, B. R., \& KEPPEL, G. An analysis of intralist similarity in verbal learning with experiments on conceptual similarity. J. verb. Learn. verb. Behav., 1965, 4, 447-462.

Note

1. A portion of this paper was previously presented at the Eastern Psychological Association meeting in Atlantic City, New Jersey, April, 1965. 